\title{
Non homogeneity of surface integrity after hard milling of high tempered bearing steel
}

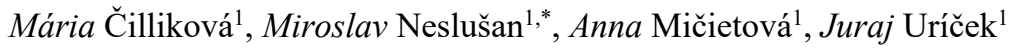 \\ ${ }^{1}$ Faculty of Mechanical Engineering, University of Zilina, 01026 Žilina, Slovakia
}

\begin{abstract}
This paper deals with surface integrity after hard milling of high tempered bearing steel $100 \mathrm{Cr} 6$. Surface integrity is expressed in terms of shape deviation as well as Barkhausen noise emission. Furthermore, components of cutting forces are also measured as a function of tool wear and their non homogenity within the contact between the milling cutter and workpiece is discussed. The results of measurements indicate that shape as well as structure remarkable non homogeneity should expected after hard milling due to missing full contact between milling cutter and workpiece. This non homogeneity can be monitored via Barkhausen noise technique. On the other hand the competitive grinding cycles produced more homogenous surface and surface state is a function of infeed speed.
\end{abstract}

Keywords: hard milling, grinding, surface integrity, Barkhausen Noise

\section{Introduction}

Cyclic magnetization in a ferromagnetic material produces magnetic pulsation as a result of irreversible and discontinuous Bloch Walls (BW) motion. Discontinuous BW motion is due to interference of BW with microstructure features such as precipitates, dislocation cells, grain boundaries and other lattice defects. This phenomenon is named Barkhausen noise (BN). BN technique has found high industrial relevance mainly for monitoring surfaces loaded near their physical limits. BN features are usually correlated with residual stresses, microhardness or structure transformations. This technique is mostly adopted for monitoring of surfaces after grinding cycles due to strong correlation among thermal over tempering, associated surface burn and the corresponding BN features [1, 2, 3, 4]. Effect of thermal softening after grinding due to over tempering decreases dislocation density (and the corresponding surface hardness), transforms carbides shape and their morphology as well as produces tensile stresses $[2,4]$. All the above mentioned aspects contribute to the higher magnitude of BN.

Nowadays, hard machining (mainly turning and milling) can substitute grinding cycles. Development in machine tools as well as in process technology raised industrial relevance of hard machining. However, hard turning or milling cycles can suffer from formation of white layers (WL) induced at low flank wear VB or unexpected catastrophic tool failures. Machined surface after hard machining is mainly a function of VB and cutting speed. Tools of high VB

\footnotetext{
* Corresponding author: miroslav.neslusan@fstroj.uniza.sk

Reviewers: Ildiko Maňková, Nataša Náprstková
} 
produce relative thick white layer (WL) as well as the corresponding heat affected zone (HAZ) $[5,6]$. On the other hand, grinding cycles can suffer from thermal over tempering. Ground surface can sometimes exhibit thermally softened layers (HAZ). However, thickness of HAZ and WL after hard milling is about 1 order lower than that induced by grinding. It is also worth to mention that the ratio between WL and HAZ thickness after hard machining is much higher as opposed to grinding $[5,7,8]$. Hard milling surfaces were already investigated and reported $[5,7,8,9]$. However significant aspect associated with non homogenity due to missing full contact between milling cutter and sample has not been discussed yet. For this reason this study is mainly focused on evaluation of surface integrity after hard milling with inserts of variable VB and compares shape deviations as well as dynamic BN signals with ground samples.

\section{Experiments and methods}

Experiments were conducted on samples made of bearing steel 100Cr6. Ten pieces of dimension 100x70x5 mm were prepared for long term test. Bearing steel was heat treated on hardness $45 \mathrm{HRC}$ (austenitizing temperature $830^{\circ} \mathrm{C}-$ oil $62^{\circ} \mathrm{C}$, followed by tempering for 2 hours temperature $470^{\circ} \mathrm{C}$ ). Cutting process was monitored as a long term test where such aspects as flank wear VB, structure alterations and corresponding surface integrity expressed in magneto elastic responses $(\mathrm{BN})$ of the hard milled surface were investigated. Cutting and other conditions: milling machine - FA4 AV, dry cutting, cutting tool made of cemented carbides R300-1240E-PM, R300-050Q22 - 12M 262489 of diameter Ø 50mm with 2 inserts of variable flank wear VB (in the range 0.05 to $0.8 \mathrm{~mm}$ ), $a_{\mathrm{p}}=0.25 \mathrm{~mm}, \mathrm{v}_{\mathrm{f}}=112 \mathrm{~mm} . \mathrm{min}^{-1}$, $\mathrm{n}=500 \mathrm{~min}^{-1}$, dry cutting. Grinding was carried out under the following conditions: grinding machine BPH 20, A9860J9V, single crystal diamond dresser, $a_{p}=0.005$ to $0.04 \mathrm{~mm}$ (10 passes), $v_{f}=8 \mathrm{~m} . \mathrm{min}^{-1}, v_{c}=25 \mathrm{~m} \cdot \mathrm{s}^{-1}$.

BN measurement was performed by the use of RollScan 300 and software package ViewScan in the frequency range of 70 to $200 \mathrm{kHz}$ (magnetizing frequency $125 \mathrm{~Hz}$, magnetizing voltage $10 \mathrm{~V}$ ). Each surface was measured in tangential direction. BN values indicated in the paper represent the effective (rms) value of BN signal. BN measurements were carried out in the dynamic mode. This means that during BN measurement components move (speed on the motion $5 \mathrm{~mm} \cdot \mathrm{s}^{-1}$ ). Cutting forces were measured by the use of three component piezoelectric dynamometer KISTLER. The signal was sampled by frequency 10 $\mathrm{kHz}$. Brief sketch of components of cutting force can be found in Fig. 1. Surface profile after machining was measured on MP 60 measuring device.

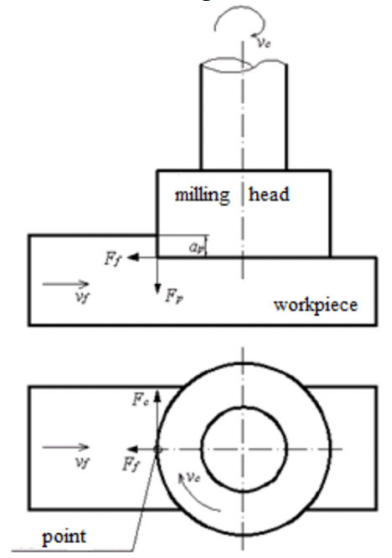

Fig. 1. Brief sketch of measurement of cutting forces during milling 


\section{Results of experiments}

Fig. 2 and Fig. 3 illustrate the typical surface which can be obtained after hard milling. The main problem is associated with the contact between milling cutter and the sample. As Fig. 2 and Fig. 3 shows that at certain position cutting inserts start to miss the full contact with the sample and run out. This position (in Fig. 2 indicated by grey color) corresponds to the cutter diameter (in this case $50 \mathrm{~mm}$ ). Due to limited toughness of the milling system milling head tends to be tilted against the machined surface in a certain small angle which changes the condition in the contact between milling cutter (cutting edge) and sample and results into the different appearance of machined surface in this zone. Fig. 3 also indicates that this zone can be easily found by visual inspection. Such character of machined surface can be found for all flank wears and the different sample hardness. Fig. 4 and also details illustrated in Fig. 5 and Fig. 6 show that this effect can be also found in the records of cutting forces components (only passive components indicated in this study, but all other components exhibit the similar behaviour). Fig. 4 and Fig. 5 show that during full contact $F_{p}$ component oscillates in the range from 200 to $700 \mathrm{~N}$ (see also Fig. 6a). As soon as the full contact is missing the run out of the insert causes fall of $F_{p}$ to zero (see also Fig. 6b). In the further process $F_{p}$ tend to decrease due to shortening path of the contact between the cutting insert and sample (see also Fig. 6c,d).

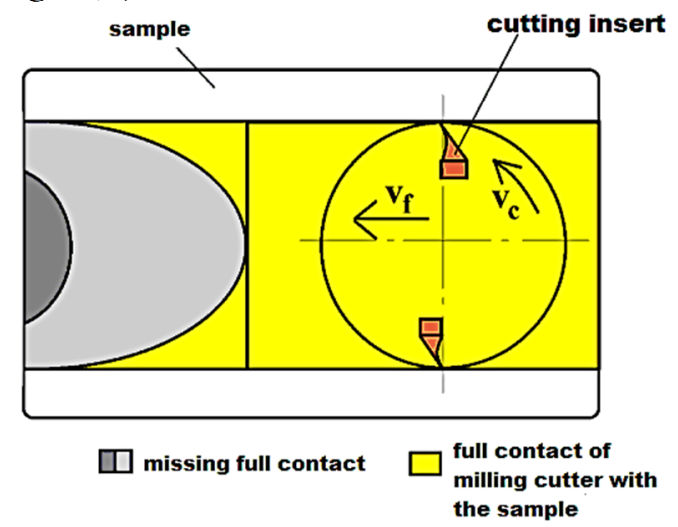

Fig. 2. Non homogenous contact between milling cutter and sample during milling

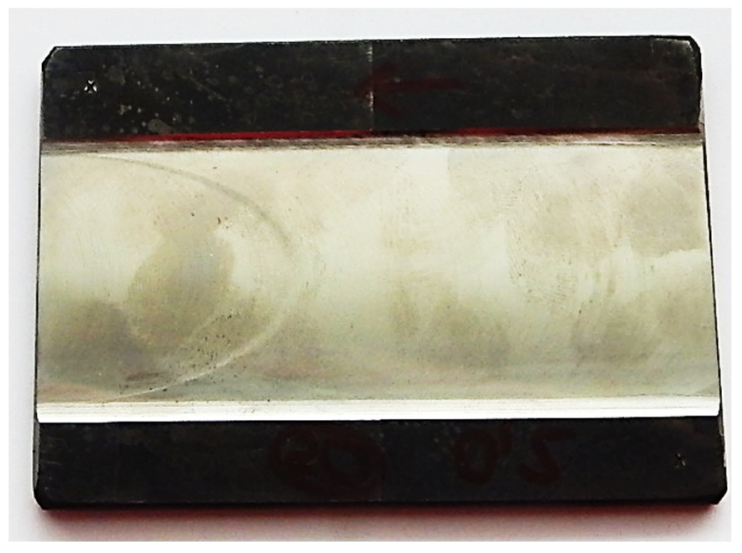

Fig. 3. Sample photo after hard milling 


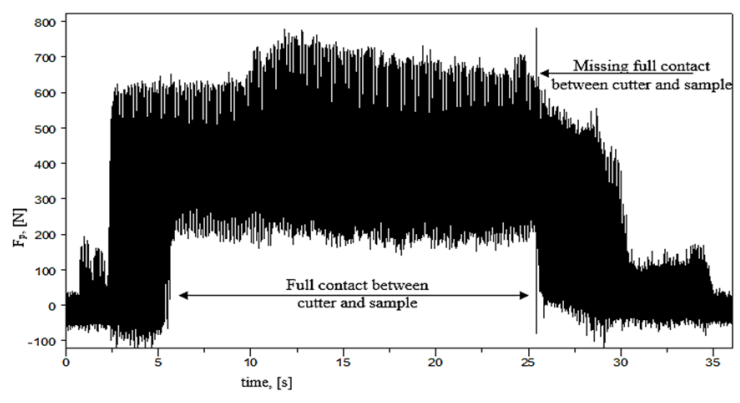

Fig. 4. Record of passive component of cutting force

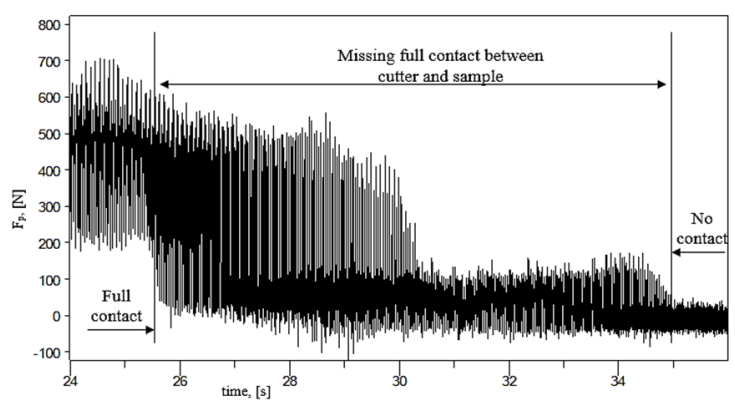

Fig. 5. Detail of passive component $F_{p}$

Further measurements of the hard milled surface produced under the variable flank wear VB indicate remarkable shape as well as structure non homogeneity of the machined surface. Fig. 7 shows record of the surface profile in which can be clearly recognized zone of full contact between the milling cutter and the sample as well as strong decrease on the surface height. This point is kept constant and corresponds to the milling cutter diameter. Quite high height of the measured profile is due to high passive forces which are typical for hard machining operation and the corresponding strong influence of passive force on precision of produced components.

Milling operations are usually employed for roughing cycles followed by consecutive final grinding. One might argue that such surface would be finished and surface non homogeneity will be removed by grinding since grinding cycles produced much better surface state from the point of view of shape deviations (see Fig. 8). However, quite high height of the profile increases allowances for the consecutive grinding cycles which in turn means longer grinding times and the corresponding costs for technological operations.

Fig. 9 shows that non homogeneity of machined surface is not only a matter of surface height (of generally surface precision expressed in any term) but also non homogeneity of microstructure and stress state should be expected since BN emission is sensitive to the alterations of microstructure expressed in all terms as well as residual stress state. Being so, it can be found that surface integrity expressed in state of microstructure as well as stress state after hard milling also exhibits remarkable non homogeneity. lace the figure as close as possible after the point where it is first referenced in the text. If there is a large number of figures and tables it might be necessary to place some before their text citation. 


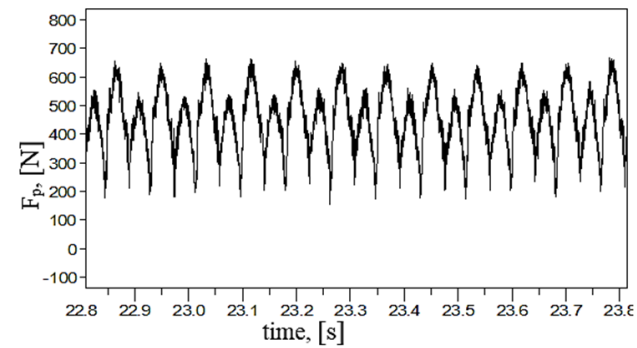

a)

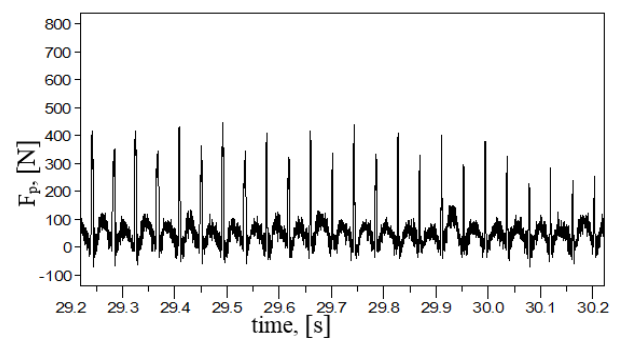

c)

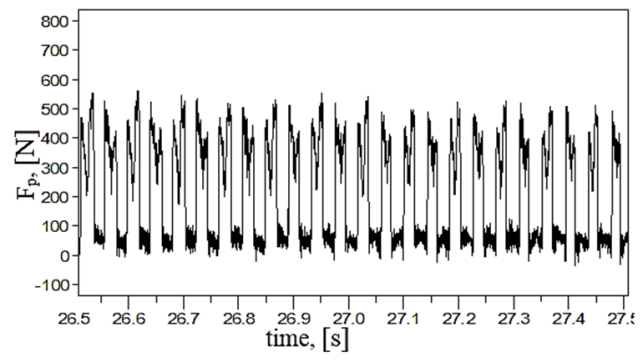

b)

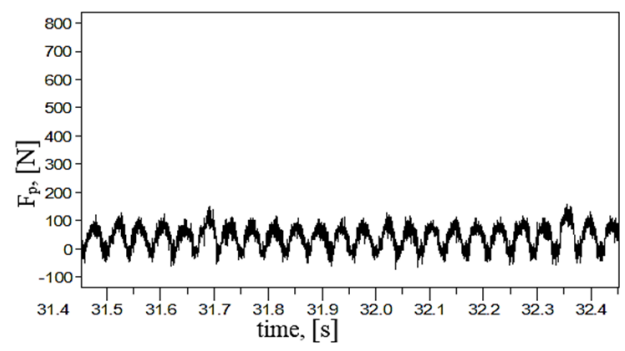

d)

Fig. 6. Details of $F_{p}$ during missing contact between milling cutter and sample - different phases

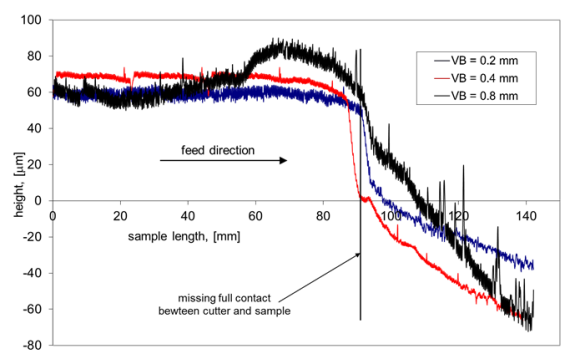

Fig. 7. Surface profile after hard milling

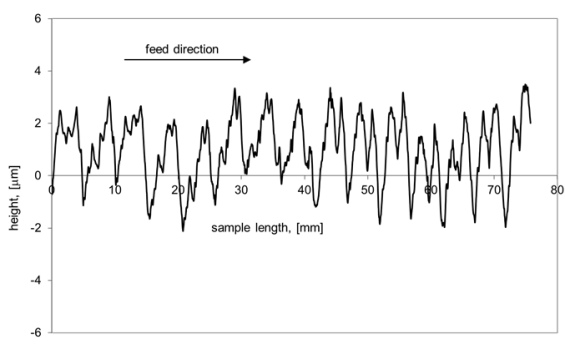

Fig. 8. Surface profile after grinding, $a_{p}=0.01 \mathrm{~mm}$

Quite similar BN values (taking into consideration 1 sample) can be found for full contact between milling cutter and the sample. As soon as full contact between milling cutter and the sample is interrupted the progressive increase or decrease of $\mathrm{BN}$ values occurs. It is worth to mention that progressive change of $\mathrm{BN}$ values in the zone of missing full contact between milling cutter and the sample correspond to the progressive change of the sample height as that illustrated in Fig. 9. On the other hand, evolution of $\mathrm{BN}$ in the zone of missing contact is not the same for all VB. As can be seen in Fig. 9, BN decreases in the feed direction in this zone for $\mathrm{VB}=0.05 \mathrm{~mm}$ whereas more or less visible increase can be found for more developed VB. The main reason can be found in the different thermal load versus VB. As is was reported, machined surface produced by insert of $\mathrm{VB}=0.05 \mathrm{~mm}$ does not contain $\mathrm{WL}$ (considering hardness $45 \mathrm{HRC}$ ). As soon as VB attains $0.4 \mathrm{~mm}$ thin WL can be found on the machined surface. It is assumed that the differences in $\mathrm{BN}$ evolution in the zone of missing contact should be associated just with this aspect.

Fig. 10 illustrates the $\mathrm{BN}$ emission and so the corresponding surface state expressed in terms of microstructure and stress state is more homogenous for grinding cycles (when compared to hard milling cycles). BN values are kept low about $200 \mathrm{mV}$ for $\mathrm{a}_{\mathrm{p}}=0.01$ and $0.02 \mathrm{~mm}$. On the other hand, increased cutting depth in grinding results into surface overheating and remarkable surface burn associated with the intensive surface oxidation 
appearing as coloured surface, see Fig. 11. Surface overheating and the corresponding surface re-hardening results into initiation of WL. As it is well known WL after grinding decreases $\mathrm{BN}$ emission and contributes to the lower BN [3] for high infeed rates as that depicted in Fig. 10. Higher infeed rates during grinding contribute to visible waviness of $\mathrm{BN}$ signal due to grinding process instability.

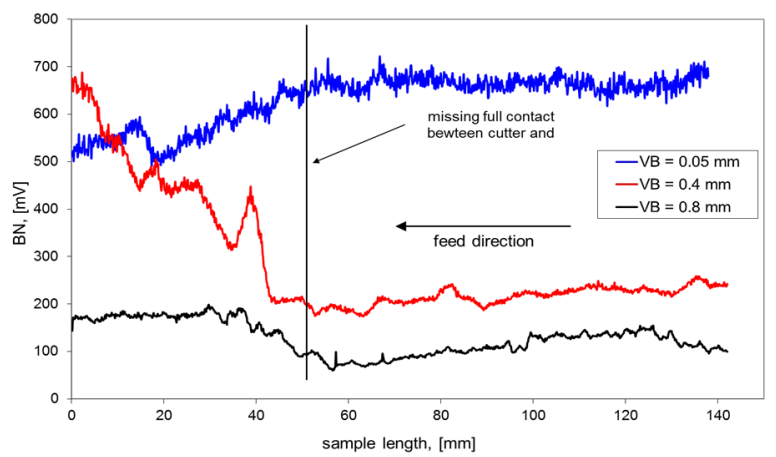

Fig. 9. Dynamic BN record after hard milling

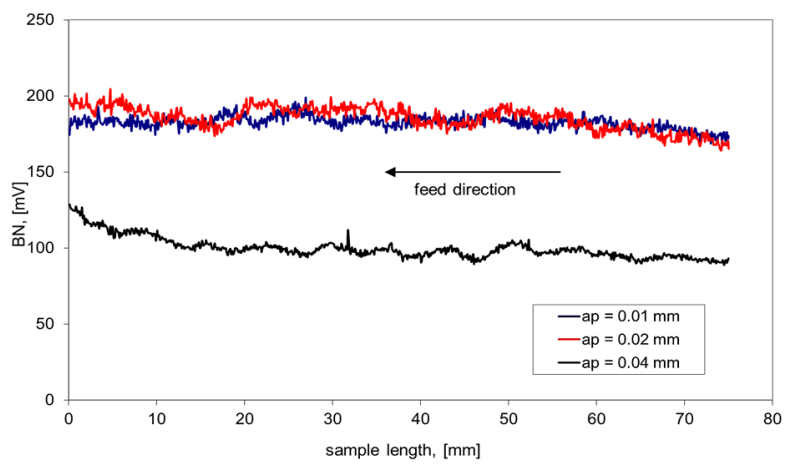

Fig. 10. Dynamic BN record after grinding

It is also worth to mention that character of WL initiated by hard milling and grinding is different. Thin WL after hard milling increases BN due to preferential orientation of matrix and corresponding BWs alignment into the direction of measurement [9]. As soon as thickness of WL after hard milling increases (along with more developed VB) BN decreases due to increasing volume of retained austenite as a non ferromagnetic feature strongly hindering BWs motion.

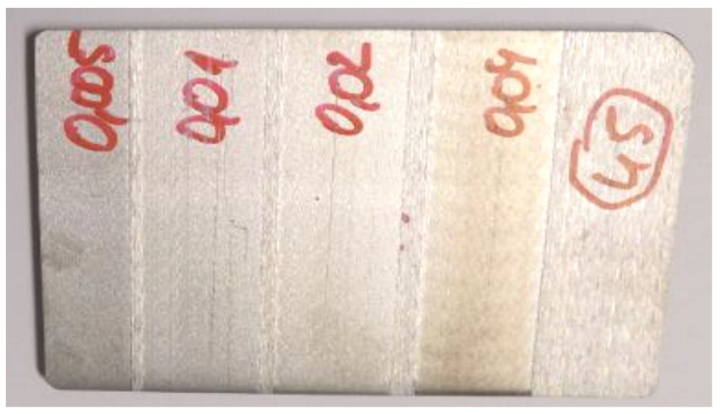

Fig. 11. Sample photo after grinding 


\section{Conclusions}

This study reports about certain disadvantages of hard milling process. Strong non homogeneity of hard milled surface can complicate the consecutive grinding cycles not only from the point of view of the allowances but also initiates complications when non destructive monitoring of the surface after grinding is carried out via $\mathrm{BN}$ technique. Industrial experience indicates that the high $\mathrm{BN}$ emission after hard milling or turning (when hard turning or milling is performed as a roughing cycle before grinding) can result in certain difficulties when surface after finishing grinding is monitored via $\mathrm{BN}$ technique. As it was mentioned, the high $\mathrm{BN}$ emission after grinding is associated with surface burn due to over-tempering. Typical thickness of the layer ground off by finishing grinding is about $0.2 \div 0.3 \mathrm{~mm}$. However, in production large bearings (or large components) some areas affected by previous hard milling or turning are not fully removed by grinding and retain below the ground surface. Being so, it could be difficult to distinguish between the high values originated from hard milling or over-tempering during grinding. As a consequence of this some parts after grinding could be referred as thermally damaged despite correctly performed finishing grinding.

This study was carried out under the financial support of VEGA agency project n. 1/0121/17 and KEGA project n.008ŽU-4/2018.

\section{References}

1. B. Karpucheewski, Introduction to micro magnetic techniques, ICBN 01, Hanover. (1998).

2. M. Mičúch, M. et all., Micro magnetic study of cutting conditions and grinding wheel wear influence on surface integrity, Manufacturing Technology, Vol. 14, 66-71 (2014)

3. V. Moorthy, et all.. Evaluation of heat treatment and deformation induced changes in material properties in gear steels using magnetic Barkhausen noise analysis, ICBN 03, Tampere, (2001)

4. M. Neslušan, J. Č́́žzek, K. Kolařík, P. Minárik, M. Čilliková, O. Melikhova, Monitoring of grinding burn via Barkhausen noise emission in case-hardened steel in large-bearing production, J. Mater. Proc. Technol., Vol. 240, 104-117. (2017)

5. Y.B. Guo, J. Sahni, A comparative study of hard turned and cylindrical ground white layers, Int. J. of Mach. Tool \& Manuf., Vol. 44, 135-145. (2004)

6. D. Brandt, D., Randzonenbeeinflussung beim Hartdrehen, PhD. Dissertation, Universität Hanover, (1995)

7. M. Neslušan, M. Rosipal, V. Ochodek, Analysis of some aspects of surface integrity after grinding and hard turning trough Barkhausen noise, ICBN 09, Hejnice. (2011).

8. M. Neslušan et. all., Application of Barkhausen noise for analysis of surface integrity after hard turning, Manufacturing technology, Vol. 12, 60-65 (2012)

9. M. Neslušan, Magnetic anisotropy in hard turned surfaces, Acta Physica Polonica A, Vol.6, 188 - 189. (2014) 